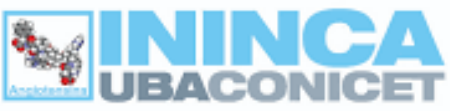

\title{
Neuroactivesteroids as neuroprotective agents against the neurodegenerative effects induced by perinatal hypoxia-ischemia injury
}

\author{
Nicolás Toro-Urrego ${ }^{1}$, Juan Pablo Luaces ${ }^{1}$, Andrea Aguilar ${ }^{1}$ and Francisco Capani ${ }^{1,2,3^{*}}$ \\ ${ }^{1}$ Instituto de Investigaciones Cardiológicas. Universidad de Buenos Aires - Consejo Nacional de \\ Investigaciones Científicas y Técnicas. ININCA. UBA-CONICET, Buenos Aires, Argentina \\ 2.Facultad de Psicología y Psicopedagogía, Universidad Católica Argentina, Buenos Aires, Argentina \\ 3.Facultad de Medicina, Fundación H. A. Barceló. \\ * Corresponding author: Franciscocapani@hotmail.com
}

Hypoxic-ischemic (HI) brain injury is one of main causes of long-term neurologic disability, morbidity and death worldwide in adults and children. This public health concern is mainly characterized by a decrease in oxygen concentration and blood flow to the tissues, which lead to cell death by energy depletion and increases free radical generation and inflammation, caused by an inefficient supply of nutrients to the brain. Hypoxic-ischemic brain injury occurs in perinatal asphyxia (PA), an obstetric complication associated with an impaired gas exchange[1]. The incidence of PA is estimated at 1/1000 live births in developed countries. Currently, there are no effective pharmaceutical strategies to prevent the triggering of secondary injury cascades, including oxidative stress and metabolic dysfunction. Neuroactive steroids like selective estrogen receptor modulators, SERMs, exert several neuroprotective effects. These include a decrease in reactive oxygen species, mitochondrial survival and maintenance of cell viability. In this context, these neurosteroids constitute promising molecules, which could modify brain response to injury, motivated us to investigate the role of Raloxifene, a selective estrogen receptor modulator in astrocytic cells under a HI injury. To replicate the conditions that occur in the presence of deprivation or decrease in $\mathrm{O} 2$ and glucose levels several studies have proposed a model of $\mathrm{O} 2$ and glucose deprivation (OGD)[1]. For this study T98G cells were seeded onto 24-wells plates in DMEM culture medium containing 10\% FBS at seeding density of 10,000 cells per well and incubated for 2-3 days until they reach confluence. Subsequently, medium was changed to glucose-free DMEM, and then incubated in $1 \% \mathrm{O} 2$ in a hypoxia incubator (Hypoxia Incubator Chamber, STEMCELL) for 9 hours. This was followed by reperfusion by changing the media to high glucose DMEM supplemented with $10 \%$ FBS and transferring the cells to $37^{\circ} \mathrm{C}$ in $95 \%$ air $/ 5 \% \mathrm{CO} 2$ in normal conditions, the control group was maintained in normal conditions of $\mathrm{O} 2$ and glucose during the experiment. For drug treatments cell cultures were incubated in DMEM serum-free medium containing 1, 0.1 and $0.01 \mathrm{uM}$ Raloxifene, as co-treatment of OGD and reperfusion. Determination of Mitochondrial Mass was evaluated using Nonyl acridine orange (NAO). NAO is a cell-permeate, cationic fluorescent dye sequestered by active mitochondria [2]. After 3 of treatments, cells were loaded in the dark with $5 \mu \mathrm{M} \mathrm{NAO}$ at $37^{\circ} \mathrm{C}$ for $20 \mathrm{~min}$. Thereafter, cells were washed with PBS to eliminate all non-sequestered dye. Mitochondrial mass was further evaluated by Fluorescence Intensity. DIC and fluorescence images were obtained with NIKON - Eclipse Ti-E PFS microscope. The analysis of cellular fluorescence was performed using Fiji [3]. The microphotographs were loaded, and background signals were eliminated from the images. Subsequently, 50 cells were randomly selected using a numbered grid in each microphotograph. The mean fluorescence value of the 50 cells was determined in eight microphotographs for each treatment using the Measure algorithm of Fiji and selecting each cell manually via ROI's (Regions of Interest) Management. There were no variations in the conditions of the image processing. Each assay was performed with a minimum of six replicate wells for each condition and experiments were repeated three times. Qualitative results suggested that 

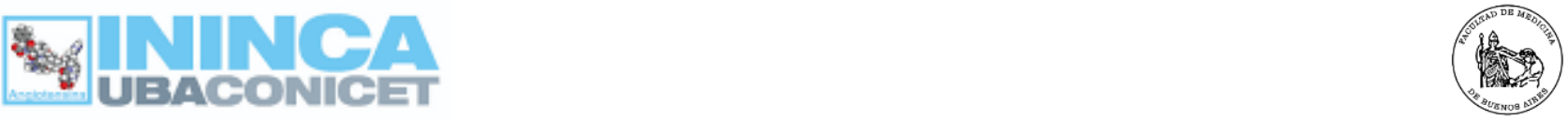

OGD may induce a loss of mitochondrial mass (Figure 1) and Raloxifene reduced this effect. Quantitative studies using fluorescence intensity for NAO confirmed these observations (Figure 2). OGD significantly reduced mitochondrial mass $(\mathrm{p}=0,0094)$ in comparison to Control group, and 0.1 uM Raloxifene significantly increased NAO fluorescence in OGD cells $(p<0,0196)$. Our results suggest that Raloxifene could be considered as a promising neuroprotective agent for HI injury.

\section{REFERENCES}

[1] N. Toro-Urrego, D. J. Vesga-Jiménez, M. I. Herrera, J. P. Luaces, and F. Capani. Curr. Neuropharmacol.17( 2018), p.874.

[2] N. Toro-Urrego, L. M. Garcia-Segura, V. Echeverria, and G. E. Barreto. Front. Aging Neurosci. 8 (2016), p. 152.

[3] J. Schindelin et al., Nat. Methods. 9 (2012), p. 676.

Figure 1. The panel shows representative microphotographs of NAO fluorescence in T98G cells exposed to 9h OGD 3h Reperfusion

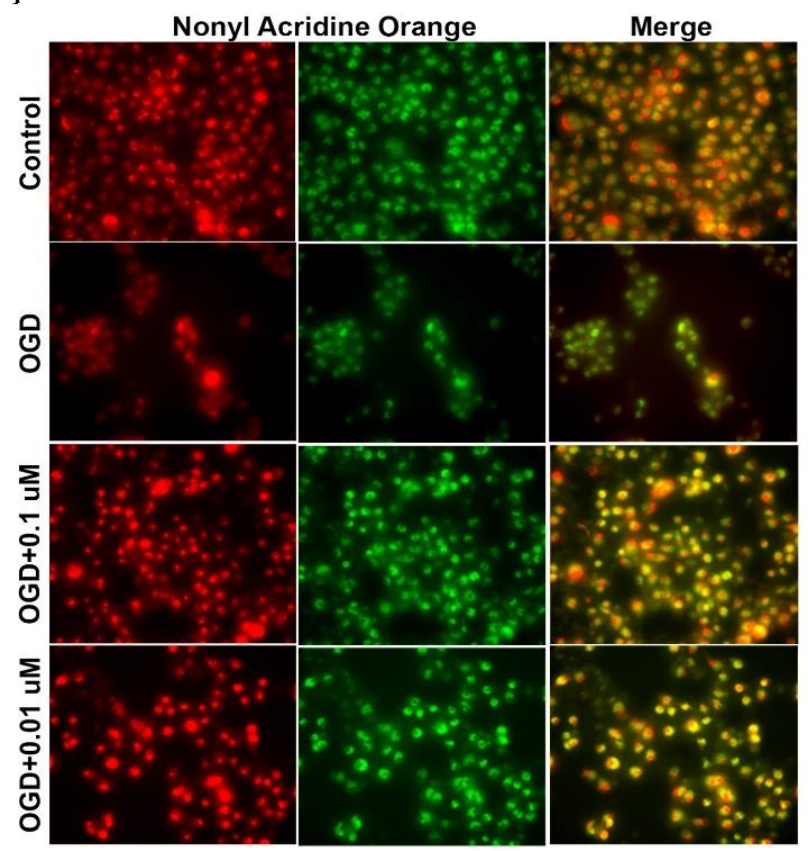

Figure 2. Raloxifene preserves mitochondrial mass. The bar graph shows the values of NAO Mean fluorescence, data are represented as Mean \pm SEM of 3 independent experiments: Control (452,2 \pm 22,28), OGD $(330,4 \pm 23,45)$, OGD+0.1 uM Raloxifene $(397,8 \pm 12,74)$, OGD+ 0.01 uM Raloxifene $(374,6 \pm 19,78)$.

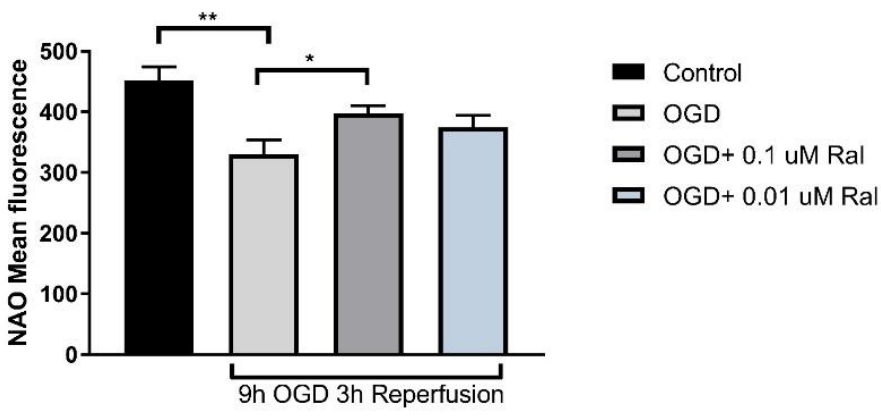

